\title{
On Some Ve-Degree and Harmonic Molecular Topological Properties of Carborundum
}

\author{
Murat Cancan ${ }^{1}$, Kerem Yamaç ${ }^{1}$, Ziyattin Taş ${ }^{2}$ and Mehmet Şerif Aldemir ${ }^{3}$ \\ ${ }^{1}$ Department of Mathematics Education, Van Yüzüncü Y1l University, \\ Zeve Campus, Tuşba, 65080, Van, Turkey \\ ${ }^{2}$ Department of Mathematics, Bingöl University, \\ Faculty of Science and Letters, Bingöl, 12000, Turkey \\ ${ }^{3}$ Department of Mathematics, Van Yüzüncü Y1l University, \\ Zeve Campus, Tuşba, 65080, Van, Turkey
}

\begin{abstract}
Carborundum, also known as silicon carbide which containing carbon and silicon, is a semiconductor. Molecular topological properties of physical substances are important tools to investigate the underlying topology of these substances. Ev-degree and ve-degree based on the molecular topological indices have been defined as parallel to their corresponding classical degree based topological indices in chemical graph theory. Classical degree based topological properties of carborundum have been investigated recently. As a continuation of these studies, in this study, we compute novel ve-degree harmonic, ve-degree sum-connectivity, ve-degree geometric-arithmetic, and ve-degree atom-bond connectivity, the first and the fifth harmonic molecular topological indices of two carborundum structures.
\end{abstract}

Index Terms - Carborundum, Harmonic indices, Ve-degree topological indices.

\section{INTRODUCTION}

This chemical graph theory study of molecules through topological indices enables to understand and to give information about underlying topology of these molecules. These studies are important part of quantitative structure \pm property relationship/quantitative structureactivity relationship studies in chemistry. Topological indices are grouped into five categories: Classical degree based topological indices such as Zagreb indices, distance based topological indices such as Wiener index, eccentric connectivity indices, classical degree, and distance based topological indices such as Gutman index, and eigenvalue based topological indices such as Estrada index and matching based topological indices such as Hosoya index. In recent

ARO-The Scientific Journal of Koya University Vol. VIII, No.1 (2020), Article ID: ARO.10560, 9 pages DOI: $10.14500 /$ aro. 10560

Received 07 August 2019; Accepted 27 April 2020

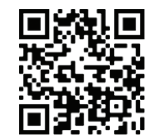

Review paper: Published 15 May 2020

Corresponding author's e-mail: mcancan@yyu.edu.tr

Copyright (C) 2020 Murat Cancan, Kerem Yamaç, Ziyattin Taş, Mehmet Şerif Aldemir.. This is an open-access article distributed under the Creative Commons Attribution License.
5 years period, studies of calculating topological indices of molecules have been rising.

Carborundum (silicon carbide), an inorganic compound has a crystal structure like that of diamond and is almost as hard. It is used as an abrasive for cutting, grinding, and polishing, as an antislip additive, and as a refractory. Carborundum is not a natural mineral, but rather is produced in a number of industrial processes. Carborundum has a wide range of industrial uses; in electronic applications including high voltage/high temperature semiconductor and grains bonded together to form extremely hard ceramics, which are used in car brakes and clutches, and plates in bulletproof vests. Stored energy release in neutron irradiated silicon carbide was investigated in (Snead, et al., 2019). 3-D Wire bondless switching cell using flip-chip-bonded silicon carbide power devices were investigated by Seal, Glover and Mantooth (2018). Reddy, Kesavan and Ramnath (2018), mechanical properties of aluminum 6061-silicon carbide, boron carbide metal matrix composite, were investigated. Real-time junction temperature sensing for silicon carbide MOSFET with different gate drive topologies and different operating conditions was studied in (Van der Broeck, et al., 2018). Effect of carbon nanoparticle reinforcement on mechanical and thermal properties of silicon carbide ceramics was studied in (Kaźmierczak-Bałata and Mazur, 2018). Topologically protected interface phonons in two-dimensional nanomaterials: Hexagonal boron nitride and silicon carbide were investigated by Jiang, Wang and Park (2018). Relaxation of residual microstress in reaction bonded silicon carbide was studied in (Wing and Halloran, 2018). Manufacturing isotropic carbon fiber preforms for multilayered silicon carbide composites with a pyrolytic carbon interphase was investigated in (Mei, et al., 2018). Ion irradiation-induced novel microstructural change in silicon carbide nanotubes was studied by Taguchi, Yamamoto and Ohba (2018).

Topological indices of nanostructures have been conducting by many researches recently in chemical graph theory such as (Ediz, 2010), (Ediz, 2011), (Ediz, 2013), (Munir, et al., 2016), (Gao, Asif and Nazeer, 2018), (Anjum and Safdar, 2019), and (Shao, et al., 2019). 
Molecular classical degree based topological properties of silicon carbon structures have been investigated recently in (Imran, et al., 2018). The authors calculated general Randic, Zagreb types indices, geometric arithmetic index, atom-bond connectivity $(\mathrm{ABC})$ index, fourth $\mathrm{ABC}$ and fifth geometric arithmetic index of $\mathrm{Si}_{2} \mathrm{C}_{3}-\mathrm{I}[\mathrm{p}, \mathrm{q}]$ and $\mathrm{Si}_{2} \mathrm{C}_{3}-\mathrm{II}[\mathrm{p}, \mathrm{q}]$. As a continuation of this study, generalized first and multiplicative Zagreb indices, the multiplicative version of the $\mathrm{ABC}$ index and the generalized multiplicative geometric arithmetic index of $\mathrm{Si}_{2} \mathrm{C}_{3}-\mathrm{I}[\mathrm{p}, \mathrm{q}]$ and $\mathrm{Si}_{2} \mathrm{C}_{3}-\mathrm{II}[\mathrm{p}, \mathrm{q}]$ have been investigated in (Kwun, et al., 2018). Reverse Zagreb and reverse hyperZagreb Indices for silicon carbide have been computed by Virk, Jhangeer and Rehman (2018).

Harmonic index was defined in (Zhong, 2012). Novel harmonic indices have been defined by Ediz, Farahani and Imran (2017). Novel ev-degree and ve-degree concepts were defined in (Chellali, et al., 2017). Moreover, novel ev-degree and ve-degree topological indices have been defined in (Ediz, 2017; Sahin and Ediz, 2018; Ediz, 2018).

The aim of this study to investigate the first, fifth, sixth harmonic, and ve-degree atom-bond connectivity (ve-ABC), geometric-arithmetic (GA), sum-connectivity, harmonic topological properties of $\mathrm{Si}_{2} \mathrm{C}_{3}-\mathrm{I}[\mathrm{p}, \mathrm{q}]$ and $\mathrm{Si}_{2} \mathrm{C}_{3}-\mathrm{II}[\mathrm{p}, \mathrm{q}]$.

\section{Preliminaries}

In this section, we give some basic and preliminary concepts which we shall use later. A graph $G=(V, E)$ consists of two nonempty sets $V$ and 2-element subsets of $V$, namely $E$. The elements of $V$ are called vertices and the elements of $E$ are called edges. For a vertex $v, \operatorname{deg}(v)$ show the number of edges that incident to $v$. The set of all vertices which adjacent to $\mathrm{v}$ is called the open neighborhood of $v$ and denoted by $N(v)$. If we add the vertex $v$ to $N(v)$, then we get the closed neighborhood of $v, N[v]$.

And now we give the definitions of ev-degree and vedegree concepts which were given in Chellali, et al. (2017).

Definition 1 (ve-degree) Let $G$ be a connected simple graph and $v \in V(G)$. The ve-degree of the vertex $v, \operatorname{deg}_{\mathrm{ve}}(\mathrm{v})$, equals the number of different edges that incident to any vertex from the closed neighborhood of $v$.

We also can restate the definition 1 as follows: Let $G$ be a connected simple graph and $v \in V(G)$. The ve-degree of the vertex $v$ is the number of different edges between the other vertices with a maximum distance of two from the vertex $v$.

Definition 2 (ev-degree), let $G$ be a connected graph and $e=u v \in E(G)$. The ev-degree of the edge $e, \operatorname{deg}_{e v}(e)$, equals the number of vertices of the union of the closed neighborhoods of $u$ and $v$.

The authors in (Chellali, et al. 2017) also can give the definition 2 as follows: Let $G$ be a connected graph and $e=u v \in E(G)$. The ev-degree of the edge $e$, $\operatorname{deg}_{u v}(e)=\operatorname{deg} u+\operatorname{deg} v-n_{e^{\prime}}$, where $n_{e}$ means the number of triangles in which the edge $e$ lies in.

Definition 3 (ve-ABC index), let $G$ be a connected graph and $e=u v \in E(G)$. The ve-ABC index of the graph $G$ defined as;

$$
A B C^{v e}(G)=\sum_{u v \in E(G)} \sqrt{\frac{\operatorname{deg}_{v e} u+\operatorname{deg}_{v e} v-2}{\operatorname{deg}_{v e} u d e g_{v e} v}}
$$

Definition 4 (ve-degree GA [ve-GA] index), let $G$ be a connected graph and $v \in V(G)$. Ve-GA index of the graph $G$ defined as;

$$
G A^{v e}(G)=\sum_{u v \in E(G)} \frac{2 \sqrt{\operatorname{deg}_{v e} u d e g_{v e} v}}{\operatorname{deg}_{v e} u+\operatorname{deg}_{v e} v}
$$

Definition 5 (ve-degree harmonic (ve-H) index), let $G$ be a connected graph and $u v \in E(G)$. ve-H index of the graph $G$ defined as;

$$
H^{v e}(G)=\sum_{u v \in E(G)} \frac{2}{\operatorname{deg}_{v e} u+\operatorname{deg}_{v e} v}
$$

Definition 6 (ve-degree sum-connectivity [ve- $\chi$ ] index), let $G$ be a connected graph and $u v \in E(G)$. Ve- $\chi$ index of the graph $G$ defined as;

$$
\chi^{v e}(G)=\sum_{u v \in E(G)}\left(\operatorname{deg}_{v e} u+\operatorname{deg}_{v e} v\right)^{-1 / 2}
$$

Let $G$ be a simple connected graph $G=(V, E)$. Harmonic indices may be defined as;

$$
H_{\text {general }}(G)=\sum_{u v \in E(G)} \frac{2}{Q_{u}+Q_{v}}
$$

where $Q_{u}$ is a unique parameter which is acquired from the vertex $u \in V(G)$.

Definition 9 (First Harmonic Index), the first kind of this Harmonic indices by considering $Q_{u}$ to be the degree of the vertex $u$ :

$$
H_{1}(G)=\sum_{u v \in E(G)} \frac{2}{d_{u}+d_{v}}
$$

Definition 10 (Second Harmonic Index), the second kind of this class can be defined by considering $Q_{u}$ to be the number $n_{u}$ of vertices of $G$ lying closer to the vertex $u$ than to the vertex $v$ for the edge $u v$ of the graph $G$ :

$$
H_{2}(G)=\sum_{u v \in E(G)} \frac{2}{n_{u}+n_{v}}
$$

Definition 11 (Third Harmonic Index), the third type of this class can be defined by considering $Q_{u}$ to be the number $\mathrm{m}_{\mathrm{u}}$ of edges of $G$ lying closer to the vertex $u$ than to the vertex $v$ for the edge $u v$ of the graph $G$ :

$$
H_{3}(G)=\sum_{u v \in E(G)} \frac{2}{m_{u}+m_{v}}
$$

Definition 12 (Fourth Harmonic Index), the fourth type of this class can be defined by considering $Q_{u}$ to be the eccentricity of the vertex $u$ :

$$
H_{4}(G)=\sum_{u v \in E(G)} \frac{2}{e_{u}+e_{v}}
$$

Definition 13 (Fifth Harmonic Index), the fifth type of this class can be defined by considering $Q_{u}$ to be the

$$
H_{5}(G)=\sum_{u v \in E(G)} \frac{2}{S_{u}+S_{v}}
$$


Definition 14 (Sixth Harmonic Index), and the sixth type of this class can be defined by considering $Q_{u}$ to be the $M_{u}=\prod_{v \in N(u)} d_{v}$

$$
H_{6}(G)=\sum_{u v \in E(G)} \frac{2}{M_{u}+M_{v}}
$$

\section{METHODS}

We use combinatorial computing method such as vertex partitioning method, edge partitioning strategy, degree counting strategy, discrete partitioning, and discrete counting strategy to compute our results.

\section{RESULTS}

In Imran, et al. (2018), two-dimensional lattices of $\mathrm{Si}_{2} \mathrm{C}_{3}-\mathrm{I}[\mathrm{p}, \mathrm{q}]$ and $\mathrm{Si}_{2} \mathrm{C}_{3}-\mathrm{II}[\mathrm{p}, \mathrm{q}]$ are given in the following Figs. 1 and 2, respectively.

Note that, two rows are being connecting, carbon atom $\mathrm{C}$ is brown and silicon atom $\mathrm{Si}$ is blue and red lines (edges) connects the upper and lower rows in Fig. $1 . \mathrm{Si}_{2} \mathrm{C}_{3}-\mathrm{I}[\mathrm{p}, \mathrm{q}]$ has $10 p g$ vertices and $15 p g-2 p-3 q$ edges.

Note that, two rows are connecting and green lines (edges) connect the upper and lower rows in Fig. 2. Furthermore, red lines show the connection between the unit cells in Fig. 2. $\mathrm{Si}_{2} \mathrm{C}_{3}-\mathrm{II}[\mathrm{p}, \mathrm{q}]$ has $10 p g$ vertices and $15 p g-2 p-3 q$ edges.

From Fig. 1, we give the edge partition of $\mathrm{Si}_{2} \mathrm{C}_{3}-\mathrm{I}[\mathrm{p}, \mathrm{q}]$ with respect to ve-degrees in the following Table I.

From Fig. 2, we give the edge partition of $\mathrm{Si}_{2} \mathrm{C}_{3}-\mathrm{II}[\mathrm{p}, \mathrm{q}]$ with respect to ve-degrees in the following Table II.

And we begin to compute topological indices for $\mathrm{Si}_{2} \mathrm{C}_{3}-\mathrm{I}[\mathrm{p}, \mathrm{q}]$.

Theorem 1

The ve-degree, the first harmonic and the fifth harmonic topological indices of the $\mathrm{Si}_{2} \mathrm{C}_{3}-\mathrm{I}[\mathrm{p}, \mathrm{q}]$ are

$$
\begin{aligned}
& A B C^{v e}\left(S i_{2} C_{3}-I\right)==(15 p q-14 p-21 q+20) x \sqrt{\frac{11}{42}}+q-1 \\
& +(p+2 q-4) x \sqrt{\frac{7}{32}}+(2 q+2) x \sqrt{\frac{2}{7}}+(4 p+2 q-7) x \sqrt{\frac{11}{42}} \\
& +(2 p+2 q-5) x \sqrt{\frac{5}{24}}+(2 p+2 q-3) x \sqrt{\frac{2}{7}}+(2 p+4 q-7) \\
& x \sqrt{\frac{5}{24}}+\sqrt{\frac{1}{2}}+\sqrt{\frac{2}{5}}+\sqrt{\frac{8}{25}}+\sqrt{\frac{9}{28}}+\sqrt{\frac{9}{30}}+\sqrt{\frac{13}{56}} \\
& G A^{v e}\left(S i_{2} C_{3}-I\right)=15 p q-12 p-17 q+17 \\
& +(2 q+2) x \frac{\sqrt{35}}{6}+(4 p+2 q-7) x \frac{2 \sqrt{42}}{13}+(2 q-2) \\
& x \frac{4 \sqrt{3}}{7}+(2 p+2 q-5) x \frac{4 \sqrt{10}}{13}+(2 p+2 q-3) \\
& x \frac{3 \sqrt{7}}{8}+(2 p+4 q-7) x \frac{12 \sqrt{2}}{17}+\frac{2 \sqrt{2}}{3} \\
& +\frac{2 \sqrt{15}}{8}+\frac{4 \sqrt{7}}{11}+\frac{2 \sqrt{30}}{11}+\frac{4 \sqrt{14}}{15}
\end{aligned}
$$

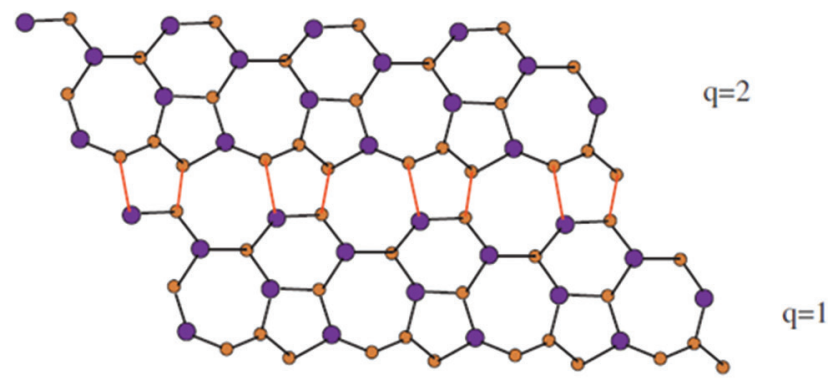

Fig. 1. Two-dimensional structure of $\mathrm{Si}_{2} \mathrm{C}_{3}-\mathrm{I}[4,2]$.

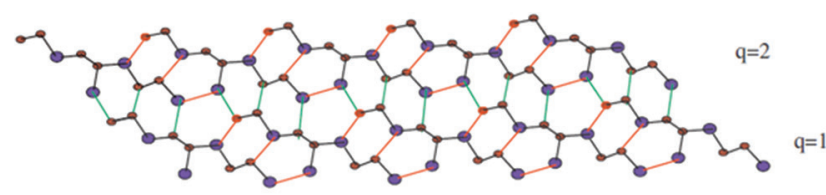

Fig. 2. Two-dimensional structure of $\mathrm{Si}_{2} \mathrm{C}_{3}-\mathrm{II}[5,2]$.

TABLE I

Ve-degrees of the END Vertices of $\mathrm{Si}_{2} \mathrm{C}_{3}-\mathrm{I}[\mathrm{P}, \mathrm{Q}]$

\begin{tabular}{lcc}
\hline \hline Edge & Ve-degrees of end vertices of edge & Number of edges \\
\hline$(2,1)$ & $(4,2)$ & 1 \\
$(3,1)$ & $(5,3)$ & 1 \\
$(2,2)$ & $(5,5)$ & $\mathrm{p}+2 \mathrm{q}$ \\
$(3,2)$ & $(5,5)$ & 1 \\
$(3,2)$ & $(7,4)$ & 1 \\
$(3,2)$ & $(7,5)$ & $2 \mathrm{q}+2$ \\
$(3,2)$ & $(5,6)$ & 1 \\
$(3,2)$ & $(7,6)$ & $4 \mathrm{p}+2 \mathrm{q}-7$ \\
$(3,2)$ & $(8,6)$ & $2 \mathrm{q}-2$ \\
$(3,2)$ & $(8,5)$ & $2 \mathrm{p}+2 \mathrm{q}-5$ \\
$(3,3)$ & $(8,7)$ & 1 \\
$(3,3)$ & $(8,8)$ & $\mathrm{p}+2 \mathrm{q}-4$ \\
$(3,3)$ & $(9,7)$ & $2 \mathrm{p}+2 \mathrm{q}-3$ \\
$(3,3)$ & $(9,8)$ & $2 \mathrm{p}+4 \mathrm{q}-7$ \\
$(3,3)$ & $(9,9)$ & $15 \mathrm{pq}-14 \mathrm{p}-21 \mathrm{q}+20$ \\
\hline \hline
\end{tabular}

TABLE II

Ve-DEgReES OF the END Vertices OF $\mathrm{Si}_{2} \mathrm{C}_{3}$-II[P,Q]

\begin{tabular}{lcc}
\hline \hline Edge & Ve-degrees of end vertices of edge & Number of edges \\
\hline$(2,1)$ & $(3,2)$ & 2 \\
$(3,1)$ & $(7,3)$ & 1 \\
$(2,2)$ & $(4,3)$ & 2 \\
$(2,2)$ & $(5,4)$ & 2 \\
$(2,2)$ & $(5,5)$ & $2 \mathrm{p}+2 \mathrm{q}-4$ \\
$(3,2)$ & $(7,5)$ & $2 \mathrm{p}+2 \mathrm{q}$ \\
$(3,2)$ & $(7,6)$ & $2 \mathrm{p}+2 \mathrm{q}-2$ \\
$(3,2)$ & $(8,6)$ & $2 \mathrm{p}+2 \mathrm{q}-6$ \\
$(3,2)$ & $(8,5)$ & $2 \mathrm{p}+2 \mathrm{q}-6$ \\
$(3,3)$ & $(8,7)$ & 2 \\
$(3,3)$ & $(8,8)$ & $2 \mathrm{p}+2 \mathrm{q}-8$ \\
$(3,3)$ & $(7,7)$ & 2 \\
$(3,3)$ & $(9,7)$ & $2 \mathrm{p}+2 \mathrm{q}-3$ \\
$(3,3)$ & $(9,8)$ & $2 \mathrm{p}+2 \mathrm{q}-5$ \\
$(3,3)$ & $(9,9)$ & $15 \mathrm{pq}-19 \mathrm{p}-19 \mathrm{q}+23$ \\
\hline \hline
\end{tabular}




$$
\begin{aligned}
& H^{v e}\left(S i_{2} C_{3}-I\right)=(15 p q-14 p-21 q+20) \\
& x \frac{1}{9}+(2 p+4 q-7) x \frac{2}{17}+(p+2 q-4) x \frac{1}{8} \\
& +(2 p+2 q-3) x \frac{1}{8}+(4 p+2 q-7) \times \frac{2}{13}+(2 q-2) \\
& \text { - } x \frac{1}{7}+(2 p+2 q-5) x \frac{2}{13}+(q+1) x \frac{1}{3}+(p+2 q+1) \\
& x \frac{1}{5}+\frac{2}{11}+\frac{1}{3}+\frac{1}{4}+\frac{2}{11}+\frac{2}{15} \\
& \chi^{v e}\left(S i_{2} C_{3}-I\right)=(15 p q-14 p-21 q+20) \\
& x \frac{1}{3 \sqrt{2}}+(2 p+4 q-7) x \frac{1}{\sqrt{17}}+(3 p+4 q-7) \\
& x \frac{1}{4}+(2 p+2 q-5) x \frac{1}{\sqrt{13}}+(2 q-2) x \frac{1}{\sqrt{14}} \\
& \text { - }+(4 p+2 q-7) x \frac{1}{\sqrt{13}}+(q+1) x \frac{1}{\sqrt{3}}+(p+2 q+1) \\
& x \frac{1}{\sqrt{10}}+\frac{1}{\sqrt{6}}+\frac{1}{2 \sqrt{2}}+\frac{2}{\sqrt{11}}+\frac{1}{\sqrt{15}} \\
& H_{1}\left(S i_{2} C_{3}-I\right)=(15 p q-9 p-13 q+9) x \frac{1}{3}+(6 p+8 q-5) \\
& \text { - } x \frac{2}{5}++(p+2 q+1) x \frac{1}{2} \\
& H_{5}\left(S i_{2} C_{3}-I\right)=(15 p q-14 p-21 q+20) \\
& x \frac{1}{9}+(2 p+4 q-7) x \frac{2}{17}+(p+2 q-4) \times \frac{1}{8} \\
& +(2 p+2 q-3) \times \frac{1}{8}+(4 p+2 q-7) \times \frac{2}{13} \\
& \text { - }+(2 q-2) \times \frac{1}{7}+(2 p+2 q-5) \times \frac{2}{13}+(q+1) \times \frac{1}{3} \\
& +(p+2 q+1) x \frac{1}{5}+\frac{2}{11}+\frac{1}{3}+\frac{1}{4}+\frac{2}{11}+\frac{2}{15}
\end{aligned}
$$

Proof of Theorem 1. From Fig. 1, Table I and the definition of ve-ABC index, we can write directly;

$$
\begin{aligned}
& A B C^{v e}\left(S i_{2} C_{3}-I\right)=\sum_{u v \in E\left(S i_{2} C_{3}-I\right)} \sqrt{\frac{\operatorname{deg}_{v e} u+\operatorname{deg}_{v e} v-2}{\operatorname{deg}_{v e} u d e g_{v e} v}}= \\
& =1 x \sqrt{\frac{4}{8}}+1 x \sqrt{\frac{6}{15}}+(p+2 q) x \sqrt{\frac{6}{15}}+1 x \sqrt{\frac{8}{25}}+1 x \sqrt{\frac{9}{28}} \\
& +(2 q+2) x \sqrt{\frac{10}{35}}+1 x \sqrt{\frac{9}{30}}+(4 p+2 q-7) x \sqrt{\frac{11}{42}} \\
& +(2 q-2) x \sqrt{\frac{12}{48}}+(2 p+2 q-5) x \sqrt{\frac{15}{72}}+1 x \sqrt{\frac{13}{56}} \\
& +(p+2 q-4) x \sqrt{\frac{14}{64}}+(2 p+2 q-3) x \sqrt{\frac{14}{63}}+(2 p+4 q-7) \\
& x \sqrt{\frac{15}{72}}+(15 p q-14 p-21 q+20) x \sqrt{\frac{11}{42}}
\end{aligned}
$$

After simplification we get;

$$
\begin{aligned}
& =(15 p q-14 p-21 q+20) x \sqrt{\frac{11}{42}} \\
& +q-1+(p+2 q-4) x \sqrt{\frac{7}{32}}+(2 q+2) x \sqrt{\frac{2}{7}} \\
& +(4 p+2 q-7) x \sqrt{\frac{11}{42}}+(2 p+2 q-5) x \sqrt{\frac{5}{24}} \\
& +(2 p+2 q-3) x \sqrt{\frac{2}{7}}+(2 p+4 q-7) x \\
& \sqrt{\frac{5}{24}}+\sqrt{\frac{1}{2}}+\sqrt{\frac{2}{5}}+\sqrt{\frac{8}{25}}+\sqrt{\frac{9}{28}}+\sqrt{\frac{9}{30}}+\sqrt{\frac{13}{56}}
\end{aligned}
$$

Again from Fig. 1, Table I and the definition of ve-GA index, we can write directly;

$$
\begin{aligned}
& G A^{v e}\left(S i_{2} C_{3}-I\right)=\sum_{u v \in E\left(S i_{2} C_{3}-I\right)} \frac{2 \sqrt{\operatorname{deg}_{v e} u d e g_{v e} v}}{\operatorname{leg}_{v e} u+\operatorname{deg}_{v e} v} \\
& =1 x \frac{2 \sqrt{8}}{6}+1 x \frac{2 \sqrt{15}}{8}+(p+2 q) x \frac{2 \sqrt{25}}{10}+1 x \frac{2 \sqrt{25}}{10} \\
& +1 x \frac{2 \sqrt{28}}{11}+(2 q+2) x \frac{2 \sqrt{35}}{12}+1 x \frac{2 \sqrt{30}}{11}+(4 p+2 q-7) \\
& x \frac{2 \sqrt{42}}{13}+(2 q-2) x \frac{2 \sqrt{48}}{14}+(2 p+2 q-5) x \frac{2 \sqrt{40}}{13} \\
& +1 x \frac{2 \sqrt{56}}{15}+(p+2 q-4) x \frac{2 \sqrt{64}}{16}+(2 p+2 q-3) x \frac{2 \sqrt{63}}{16} \\
& +(2 p+4 q-7) x \frac{2 \sqrt{72}}{17}+(15 p q-14 p-21 q+20) x \frac{2 \sqrt{81}}{18} \\
& \quad=15 p q-14 p-21 q+20+p+2 q-4+p+2 q \\
& +(2 q+2) x \frac{\sqrt{35}}{6}+(4 p+2 q-7) x \frac{2 \sqrt{42}}{13}+(2 q-2) \\
& +1+\frac{4 \sqrt{7}}{11}+\frac{2 \sqrt{30}}{11}+\frac{4 \sqrt{14}}{15} \\
& +(2 p+4 q \\
& +7
\end{aligned}
$$

After simplification, we get it;

$$
\begin{aligned}
& =15 p q-12 p-17 q+17+(2 q+2) x \frac{\sqrt{35}}{6}+(4 p+2 q-7) \\
& x \frac{2 \sqrt{42}}{13}+(2 q-2) x \frac{4 \sqrt{3}}{7}+(2 p+2 q-5) x \frac{4 \sqrt{10}}{13} \\
& +(2 p+2 q-3) x \frac{3 \sqrt{7}}{8}+(2 p+4 q-7) x \frac{12 \sqrt{2}}{17} \\
& +\frac{2 \sqrt{2}}{3}+\frac{2 \sqrt{15}}{8}+\frac{4 \sqrt{7}}{11}+\frac{2 \sqrt{30}}{11}+\frac{4 \sqrt{14}}{15}
\end{aligned}
$$

Now, we prove the ve-H index of $\mathrm{Si}_{2} \mathrm{C}_{3}$-I. From Fig. 1, Table I and the definition of ve-H index, we can write directly; 


$$
\begin{gathered}
H^{v e}\left(S i_{2} C_{3}-I\right)=\sum_{u v \in E\left(S i_{2} C_{3}-I\right)} \frac{2}{d e g_{v e} u+d e g_{v e} v} \\
=1 x \frac{2}{6}+1 x \frac{2}{8}+(p+2 q) x \frac{2}{10}+1 x \frac{2}{10}+1 x \frac{2}{11}+(2 q+2) x \frac{2}{12} \\
+1 x \frac{2}{11}+(4 p+2 q-7) x \frac{2}{13}+(2 q-2) x \frac{2}{14}+(2 p+2 q-5) \\
x \frac{2}{13}+1 x \frac{2}{15}+(p+2 q-4) x \frac{2}{16}+(2 p+2 q-3) x \frac{2}{16}+\left(\begin{array}{l}
2 p+ \\
4 q-7
\end{array}\right) \\
x \frac{2}{17}+(15 p q-14 p-21 q+20) x \frac{2}{18} \\
=\frac{1}{3}+\frac{1}{4}+(p+2 q+1) x \frac{1}{5}+\frac{2}{11}+(q+1) x \frac{1}{3}+\frac{2}{11} \\
+(4 p+2 q-7) x \frac{2}{13}+(2 q-2) x \frac{1}{7}+(2 p+2 q-5) \\
\quad x \frac{2}{13}+\frac{2}{15}+(p+2 q-4) x \frac{1}{8}+(2 p+2 q-3) x \frac{1}{8} \\
+(2 p+4 q-7) x \frac{2}{17}+(15 p q-14 p-21 q+20) x \frac{1}{9}
\end{gathered}
$$

After some simplifications, we get;

$$
\begin{aligned}
& =(15 p q-14 p-21 q+20) \times \frac{1}{9}+(2 p+4 q-7) \times \frac{2}{17} \\
& +(p+2 q-4) \times \frac{1}{8}+(2 p+2 q-3) \times \frac{1}{8}+(4 p+2 q-7) \\
& x \frac{2}{13}+(2 q-2) x \frac{1}{7}+(2 p+2 q-5) \times \frac{2}{13}+(q+1) \times \frac{1}{3} \\
& +(p+2 q+1) \times \frac{1}{5}+\frac{2}{11}+\frac{1}{3}+\frac{1}{4}+\frac{2}{11}+\frac{2}{15}
\end{aligned}
$$

Now, we prove the ve- $\chi$ index of $\mathrm{Si}_{2} \mathrm{C}_{3}$-I. From Fig. 1, Table I and the definition of ve- $\chi$ index, we can write directly;

$$
\begin{aligned}
& \chi^{v e}\left(S i_{2} C_{3}-I\right)=\sum_{u v \in E\left(S i_{2} C_{3}-I\right)}\left(\operatorname{deg}_{v e} u+\operatorname{deg}_{v e} v\right)^{-1 / 2} \\
& =1 x \frac{1}{\sqrt{6}}+1 x \frac{1}{\sqrt{8}}+(p+2 q) x \frac{1}{\sqrt{10}}+1 x \frac{1}{\sqrt{10}}+1 x \frac{1}{\sqrt{11}} \\
& +(2 q+2) x \frac{1}{\sqrt{12}}+1 x \frac{1}{\sqrt{11}}+(4 p+2 q-7) x \frac{1}{\sqrt{13}} \\
& +(2 q-2) x \frac{1}{\sqrt{14}}+(2 p+2 q-5) x \frac{1}{\sqrt{13}}+1 x \frac{1}{\sqrt{15}} \\
& +(p+2 q-4) x \frac{1}{\sqrt{16}}+(2 p+2 q-3) x \frac{1}{\sqrt{16}}+(2 p+4 q-7) \\
& x \frac{1}{\sqrt{17}}+(15 p q-14 p-21 q+20) x \frac{1}{\sqrt{18}} \\
& \quad \frac{1}{\sqrt{6}}+\frac{1}{2 \sqrt{2}}+(p+2 q+1) x \frac{1}{\sqrt{10}}+\frac{2}{\sqrt{11}}+(q+1) \\
& \quad x \frac{1}{\sqrt{3}}+(4 p+2 q-7) x \frac{1}{\sqrt{13}}+(2 q-2) x \frac{1}{\sqrt{14}} \\
& +(2 p+2 q-5) x \frac{1}{\sqrt{13}}+\frac{1}{\sqrt{15}}+(3 p+4 q-7) x \frac{1}{4} \\
& \quad+(2 p+4 q-7) x \frac{1}{\sqrt{17}}+(15 p q-14 p-21 q+20) x \frac{1}{3 \sqrt{2}}
\end{aligned}
$$

After necessary simplifications, we get;

$$
\begin{aligned}
& =(15 p q-14 p-21 q+20) \times \frac{1}{3 \sqrt{2}}+(2 p+4 q-7) \\
& x \frac{1}{\sqrt{17}}+(3 p+4 q-7) x \frac{1}{4}+(2 p+2 q-5) x \frac{1}{\sqrt{13}} \\
& +(2 q-2) x \frac{1}{\sqrt{14}}+(4 p+2 q-7) \times \frac{1}{\sqrt{13}}+(q+1) \\
& x \frac{1}{\sqrt{3}}+(p+2 q+1) x \frac{1}{\sqrt{10}}+\frac{1}{\sqrt{6}}+\frac{1}{2 \sqrt{2}}+\frac{2}{\sqrt{11}}+\frac{1}{\sqrt{15}}
\end{aligned}
$$

Now, we prove the first harmonic index of $\mathrm{Si}_{2} \mathrm{C}_{3}$-I. From Fig. 1, Table I and the definition of the first harmonic index, we can write directly;

$$
\begin{gathered}
H_{1}\left(\mathrm{Si}_{2} \mathrm{C}_{3}-I\right)=\sum_{u v \in E\left(G S i_{2} C_{3}-I\right)} \frac{2}{d_{u}+d_{v}} \\
=1 x \frac{2}{3}+1 x \frac{2}{4}+(p+2 q) x \frac{2}{4}+1 x \frac{2}{5}+1 x \frac{2}{5}+(2 q+2) \\
x \frac{2}{5}+1 x \frac{2}{5}+(4 p+2 q-7) x \frac{2}{5}+(2 q-2) x \frac{2}{5}+\left(\begin{array}{l}
2 p+ \\
2 q-5
\end{array}\right) \times \frac{2}{5} \\
+1 x \frac{2}{6}+(p+2 q-4) x \frac{2}{6}+(2 p+2 q-3) x \frac{2}{6} \\
+(2 p+4 q-7) x \frac{2}{6}+(15 p q-14 p-21 q+20) \times \frac{2}{6}
\end{gathered}
$$

After necessary simplifications, we can write that;

$$
\begin{aligned}
& =\frac{2}{3}+\frac{1}{2}+(p+2 q) x \frac{1}{2}+\frac{2}{5}+\frac{2}{5}+(2 q+2) x \frac{2}{5}+\frac{2}{5} \\
& +(4 p+2 q-7) x \frac{2}{5}+(2 q-2) x \frac{2}{5}+(2 p+2 q-5) \times \frac{2}{5} \\
& +\frac{1}{3}+(p+2 q-4) x \frac{1}{3}+(2 p+2 q-3) x \frac{1}{3}+\left(\begin{array}{l}
2 p+4 q \\
-7
\end{array}\right) \\
& x \frac{1}{3}+(15 p q-14 p-21 q+20) \times \frac{1}{3} \\
& =(15 p q-9 p-13 q+9) \times \frac{1}{3}+(6 p+8 q-5) \times \frac{2}{5}+ \\
& +(p+2 q+1) \times \frac{1}{2}
\end{aligned}
$$

And now, we prove the fifth harmonic index of $\mathrm{Si}_{2} \mathrm{C}_{3}-\mathrm{I}$. From Fig. 1, Table I and the definition of the fifth harmonic index, we can write directly;

$$
\begin{gathered}
H_{5}\left(S i_{2} C_{3}-I\right)=\sum_{u v \in E\left(S i_{2} C_{3}-I\right)} \frac{2}{S_{u}+S_{v}} \\
=1 x \frac{2}{6}+1 x \frac{2}{8}+(p+2 q) x \frac{2}{10}+1 x \frac{2}{10}+1 x \frac{2}{11}+(2 q+2) \\
x \frac{2}{12}+1 x \frac{2}{11}+(4 p+2 q-7) x \frac{2}{13}+(2 q-2) x \frac{2}{14}+\left(\begin{array}{l}
2 p \\
+2 q-5
\end{array}\right) \\
x \frac{2}{13}+1 x \frac{2}{15}+(p+2 q-4) x \frac{2}{16}+(2 p+2 q-3) x \frac{2}{16} \\
+(2 p+4 q-7) x \frac{2}{17}+(15 p q-14 p-21 q+20) \times \frac{2}{18}
\end{gathered}
$$




$$
\begin{aligned}
& =\frac{1}{3}+\frac{1}{4}+(p+2 q+1) x \frac{1}{5}+\frac{2}{11}+(q+1) \times \frac{1}{3} \\
& +\frac{2}{11}+(4 p+2 q-7) x \frac{2}{13}+(2 q-2) \times \frac{1}{7} \\
& +(2 p+2 q-5) x \frac{2}{13}+\frac{2}{15}+(p+2 q-4) \times \frac{1}{8} \\
& +(2 p+2 q-3) x \frac{1}{8}+(2 p+4 q-7) \\
& x \frac{2}{17}+(15 p q-14 p-21 q+20) \times \frac{1}{9}
\end{aligned}
$$

After necessary simplifications, we can write that;

$$
\begin{aligned}
& =(15 p q-14 p-21 q+20) \times \frac{1}{9} \\
& +(2 p+4 q-7) x \frac{2}{17}+(p+2 q-4) \\
& x \frac{1}{8}+(2 p+2 q-3) x \frac{1}{8}+(4 p+2 q-7) x \frac{2}{13} \\
& +(2 q-2) x \frac{1}{7}+(2 p+2 q-5) x \frac{2}{13}+(q+1) x \frac{1}{3} \\
& +(p+2 q+1) x \frac{1}{5}+\frac{2}{11}+\frac{1}{3}+\frac{1}{4}+\frac{2}{11}+\frac{2}{15}
\end{aligned}
$$

And now, we begin to compute topological indices for $\mathrm{Si}_{2} \mathrm{C}_{3}-I I[p, q]$.

Theorem 2

The ve-degree, the first and the fifth harmonic topological indices of the $\mathrm{Si}_{2} \mathrm{C}_{3}-\mathrm{II}[\mathrm{p}, \mathrm{q}]$ are

$$
\begin{aligned}
& A B C^{v e}\left(S i_{2} C_{3}-I I\right)=(15 p q-19 p-19 q+23) \\
& x \frac{4}{9}+p+q-3+(2 p+2 q-5) x \sqrt{\frac{5}{24}} \\
& +(2 p+2 q-3) x \sqrt{\frac{14}{63}}+(2 p+2 q-8) x \sqrt{\frac{7}{32}} \\
& \text { - }+(2 p+2 q-6) x \sqrt{\frac{11}{40}}+(2 p+2 q-2) x \sqrt{\frac{11}{42}}+ \\
& +(2 p+2 q) x \sqrt{\frac{10}{35}}+(2 p+2 q-4) x \sqrt{\frac{8}{25}}+\sqrt{2}+\sqrt{\frac{8}{21}} \\
& +2 x \sqrt{\frac{5}{12}}+\sqrt{\frac{7}{5}}+\sqrt{\frac{13}{14}}+4 x \sqrt{\frac{3}{49}} \\
& G A^{v e}\left(S i_{2} C_{3}-I I\right)=15 p q-15 p-15 q+(2 p+2 q) \\
& x \frac{2 \sqrt{35}}{12}+(2 p+2 q-2) x \frac{2 \sqrt{42}}{13}+(2 p+2 q-6) \\
& x \frac{4 \sqrt{3}}{7}+(2 p+2 q-6) x \frac{8 \sqrt{10}}{13}+(2 p+2 q-3) \\
& x \frac{6 \sqrt{7}}{16}+(2 p+2 q-5) x \frac{12 \sqrt{2}}{17}+13+\frac{4 \sqrt{6}}{5} \\
& +\frac{\sqrt{21}}{5}+\frac{8 \sqrt{2}}{7}+\frac{8 \sqrt{5}}{9}+\frac{16 \sqrt{7}}{15}
\end{aligned}
$$

$$
\begin{aligned}
& H^{v e}\left(S i_{2} C_{3}-I I\right)=\frac{4}{5}+\frac{1}{5}+\frac{6}{7}+\frac{4}{9}+(2 p+2 q-4) \times \frac{1}{5} \\
& +(p+q) x \frac{1}{3}+(2 p+2 q-2) \times \frac{2}{13}+\left(\begin{array}{l}
2 p \\
+2 q-6
\end{array}\right) x \frac{1}{7}+\left(\begin{array}{l}
2 p \\
+2 q-6
\end{array}\right) \\
& x \frac{2}{13}+\frac{4}{15}+\left(\begin{array}{l}
2 p \\
+2 q-8
\end{array}\right) x \frac{1}{8}+\left(\begin{array}{l}
2 p \\
+2 q-3
\end{array}\right) x \frac{1}{8}+\left(\begin{array}{l}
2 p \\
+2 q-5
\end{array}\right) \times \frac{2}{17} \\
& +(15 p q-19 p-19 q+23) \times \frac{1}{9} \\
& \chi^{v e}\left(S i_{2} C_{3}-I I\right)=\frac{2}{\sqrt{5}}+\frac{1}{\sqrt{10}}+\frac{2}{\sqrt{7}}+\frac{2}{3}+\frac{2}{\sqrt{15}}+\frac{2}{\sqrt{14}} \\
& +\left(\begin{array}{l}
2 p+2 q \\
-4
\end{array}\right) x \frac{1}{\sqrt{10}}+(p+q) x \frac{1}{\sqrt{3}}+\left(\begin{array}{l}
2 p+2 q \\
-2
\end{array}\right) x \frac{1}{\sqrt{13}} \\
& \text { - }+\left(\begin{array}{l}
2 p+ \\
2 q-6
\end{array}\right) x \frac{1}{\sqrt{14}}+(2 p+2 q-6) x \frac{1}{\sqrt{13}}+\left(\begin{array}{l}
p+q \\
-4
\end{array}\right) x \frac{1}{2} \\
& +\left(\begin{array}{l}
2 p+ \\
2 q-3
\end{array}\right) x \frac{1}{\sqrt{16}}+\left(\begin{array}{l}
2 p+ \\
2 q-5
\end{array}\right) x \frac{2}{\sqrt{17}}+\left(\begin{array}{c}
15 p q-19 p \\
-19 q+23
\end{array}\right) \times \frac{1}{3 \sqrt{2}} \\
& H_{1}\left(S i_{2} C_{3}-I I\right)=5+p+q+(8 p+8 q-14) x \frac{2}{5} \\
& +(15 p q-13 p-13 q+9) \times \frac{1}{3} \\
& H_{5}\left(G S i_{2} C_{3}-I I\right)=\frac{4}{5}+\frac{1}{5}+\frac{6}{7}+\frac{4}{9}+\left(\begin{array}{l}
2 p \\
+2 q-4
\end{array}\right) \times \frac{1}{5} \\
& +(p+q) x \frac{1}{3}+\left(\begin{array}{l}
2 p \\
+2 q-2
\end{array}\right) x \frac{2}{13}+\left(\begin{array}{l}
2 p \\
+2 q-6
\end{array}\right) x \frac{1}{7}+\left(\begin{array}{l}
2 p \\
+2 q-6
\end{array}\right) \\
& x \frac{2}{13}+\frac{4}{15}+\left(\begin{array}{l}
2 p \\
+2 q-8
\end{array}\right) x \frac{1}{8}+\left(\begin{array}{l}
2 p \\
+2 q-3
\end{array}\right) x \frac{1}{8}+\left(\begin{array}{l}
2 p \\
+2 q-5
\end{array}\right) \times \frac{2}{17} \\
& +(15 p q-19 p-19 q+23) \times \frac{1}{9}
\end{aligned}
$$

Proof of Theorem 2

From Fig. 2, Table II and the definition of ve-ABC index, we can write directly;

$$
\begin{aligned}
& A B C^{v e}\left(S i_{2} C_{3}-I I\right)=\sum_{u v \in E\left(S i_{2} C_{3}-I\right)} \sqrt{\frac{\operatorname{deg}_{v e} u+\operatorname{deg}_{v e} v-2}{\operatorname{leg}_{v e} u d e g_{v e} v}} \\
& =2 x \sqrt{\frac{3}{6}}+1 x \sqrt{\frac{8}{21}}+2 x \sqrt{\frac{5}{12}}+2 x \sqrt{\frac{7}{20}}+(2 p+2 q-4) \\
& \quad x \sqrt{\frac{8}{25}}+(2 p+2 q) x \sqrt{\frac{10}{35}}+(2 p+2 q-2) x \sqrt{\frac{11}{42}} \\
& +(2 p+2 q-6) x \sqrt{\frac{12}{48}}+(2 p+2 q-6) x \sqrt{\frac{11}{40}}+2 x \sqrt{\frac{13}{56}} \\
& +(2 p+2 q-8) x \sqrt{\frac{14}{64}}+2 x \sqrt{\frac{12}{49}}+\left(\begin{array}{l}
2 p \\
+2 q-3
\end{array}\right) x \sqrt{\frac{14}{63}} \\
& +(2 p+2 q-5) x \sqrt{\frac{15}{72}}+\left(\begin{array}{l}
15 p q-19 p \\
-19 q+23
\end{array}\right) x \sqrt{\frac{16}{81}}
\end{aligned}
$$


After necessary simplifications we get;

$$
\begin{aligned}
& =(15 p q-19 p-19 q+23) x \frac{4}{9}+p+q-3+(2 p+2 q-5) \\
& x \sqrt{\frac{5}{24}}+(2 p+2 q-3) x \sqrt{\frac{14}{63}}+(2 p+2 q-8) x \sqrt{\frac{7}{32}} \\
& +(2 p+2 q-6) x \sqrt{\frac{11}{40}}+(2 p+2 q-2) x \sqrt{\frac{11}{42}} \\
& +(2 p+2 q) x \sqrt{\frac{10}{35}}+(2 p+2 q-4) x \sqrt{\frac{8}{25}}+\sqrt{2} \\
& +\sqrt{\frac{8}{21}}+2 x \sqrt{\frac{5}{12}}+\sqrt{\frac{7}{5}}+\sqrt{\frac{13}{14}}+4 x \sqrt{\frac{3}{49}}
\end{aligned}
$$

And now, we prove the ve-GA index of $\mathrm{Si}_{2} \mathrm{C}_{3}$-II. From Fig. 2, Table II and the definition of ve-GA index, we can write directly;

$$
\begin{gathered}
G A^{v e}\left(S i_{2} C_{3}-I I\right)=\sum_{u v \in E\left(S i_{2} C_{3}-I I\right)} \frac{2 \sqrt{\operatorname{deg}_{v e} u d e g_{v e} v}}{\operatorname{deg}_{v e} u+\operatorname{deg}_{v e} v} \\
=15 p q-19 p-19 q+23+2+2 p+2 q-8+2 p+2 q-4 \\
+\frac{4 \sqrt{6}}{5}+\frac{\sqrt{21}}{5}+\frac{8 \sqrt{2}}{7}+\frac{8 \sqrt{5}}{9}+(2 p+2 q) x \frac{2 \sqrt{35}}{12}+\left(\begin{array}{l}
2 p \\
+2 q-2
\end{array}\right) \\
x \frac{2 \sqrt{42}}{13}+(2 p+2 q-6) x \frac{4 \sqrt{3}}{7}+(2 p+2 q-6) x \frac{8 \sqrt{10}}{13} \\
+\frac{16 \sqrt{7}}{15}+(2 p+2 q-3) x \frac{6 \sqrt{7}}{16}+(2 p+2 q-5) x \frac{12 \sqrt{2}}{17}
\end{gathered}
$$

After necessary simplifications, we can write that;

$$
\begin{aligned}
& =15 p q-15 p-15 q+(2 p+2 q) x \frac{2 \sqrt{35}}{12}+(2 p+2 q-2) \\
& x \frac{2 \sqrt{42}}{13}+(2 p+2 q-6) x \frac{4 \sqrt{3}}{7}+(2 p+2 q-6) x \frac{8 \sqrt{10}}{13} \\
& +(2 p+2 q-3) x \frac{6 \sqrt{7}}{16}+(2 p+2 q-5) x \frac{12 \sqrt{2}}{17} \\
& +13+\frac{4 \sqrt{6}}{5}+\frac{\sqrt{21}}{5}+\frac{8 \sqrt{2}}{7}+\frac{8 \sqrt{5}}{9}+\frac{16 \sqrt{7}}{15}
\end{aligned}
$$

And now, we prove the ve-H index of $\mathrm{Si}_{2} \mathrm{C}_{3}$-II. From Fig. 2, Table II and the definition of ve- $\mathrm{H}$ index, we can write directly;

$$
\begin{aligned}
& H^{v e}\left(\mathrm{Si}_{2} \mathrm{C}_{3}-I I\right)=\sum_{u v \in E\left(S_{2} C_{3}-I I\right)} \frac{2}{\operatorname{deg}_{v e} u+\operatorname{deg}_{v e} v} \\
= & 2 x \frac{2}{5}+1 x \frac{2}{10}+2 x \frac{2}{7}+2 x \frac{2}{9}+(2 p+2 q-4) \times \frac{2}{10} \\
+ & (2 p+2 q) \times \frac{2}{12}+(2 p+2 q-2) \times \frac{2}{13}+(2 p+2 q-6) \times \frac{2}{14} \\
+ & (2 p+2 q-6) x \frac{2}{13}+2 x \frac{2}{15}+(2 p+2 q-8) \times \frac{2}{16} \\
+ & 2 x \frac{2}{14}+(2 p+2 q-3) \times \frac{2}{16}+(2 p+2 q-5) \times \frac{2}{17} \\
+ & (15 p q-19 p-19 q+23) \times \frac{2}{18}
\end{aligned}
$$

After necessary simplifications, we can write that,

$$
\begin{aligned}
& =\frac{4}{5}+\frac{1}{5}+\frac{6}{7}+\frac{4}{9}+(2 p+2 q-4) \times \frac{1}{5}+(p+q) \times \frac{1}{3} \\
& +(2 p+2 q-2) x \frac{2}{13}+(2 p+2 q-6) x \frac{1}{7}+(2 p+2 q-6) \\
& x \frac{2}{13}+\frac{4}{15}+(2 p+2 q-8) x \frac{1}{8}+(2 p+2 q-3) \times \frac{1}{8}+\left(\begin{array}{l}
2 p \\
+2 q-5
\end{array}\right) \\
& x \frac{2}{17}+(15 p q-19 p-19 q+23) \times \frac{1}{9}
\end{aligned}
$$

And now, we prove the ve- $\chi$ index of $\mathrm{Si}_{2} \mathrm{C}_{3}$-II. From Fig. 2, Table II and the definition of ve- $\chi$ index, we can write directly;

$$
\begin{aligned}
\chi^{v e}( & \left.S i_{2} C_{3}-I I\right)=\sum_{u v \in E\left(G S i_{2} C_{3}-I I\right)}\left(\operatorname{deg}_{v e} u+\operatorname{deg}_{v e} v\right)^{-1 / 2} \\
= & 2 x \frac{1}{5}+1 x \frac{1}{10}+2 x \frac{1}{7}+2 x \frac{1}{9}+(2 p+2 q-4) x \frac{1}{10} \\
& +(2 p+2 q) x \frac{1}{12}+(2 p+2 q-2) x \frac{1}{13}+(2 p+2 q-6) \\
& x \frac{1}{14}+(2 p+2 q-6) x \frac{1}{13}+2 x \frac{1}{15}+(2 p+2 q-8) \\
& x \frac{1}{16}+2 x \frac{1}{14}+(2 p+2 q-3) x \frac{1}{16}+(2 p+2 q-5) \\
& x \frac{1}{17}+(15 p q-19 p-19 q+23) x \frac{1}{18}
\end{aligned}
$$

After some necessary simplifications, we can directly write that;

$$
\begin{aligned}
& =\frac{2}{\sqrt{5}}+\frac{1}{\sqrt{10}}+\frac{2}{\sqrt{7}}+\frac{2}{3}+\frac{2}{\sqrt{15}}+\frac{2}{\sqrt{14}}+(2 p+2 q-4) \\
& x \frac{1}{\sqrt{10}}+(p+q) x \frac{1}{\sqrt{3}}+(2 p+2 q-2) x \frac{1}{\sqrt{13}} \\
& +(2 p+2 q-6) x \frac{1}{\sqrt{14}}+(2 p+2 q-6) \times \frac{1}{\sqrt{13}} \\
& +(p+q-4) x \frac{1}{2}+(2 p+2 q-3) x \frac{1}{\sqrt{16}}+(2 p+2 q-5) \\
& x \frac{2}{\sqrt{17}}+(15 p q-19 p-19 q+23) x \frac{1}{3 \sqrt{2}}
\end{aligned}
$$

And now, we prove the first harmonic index of $\mathrm{Si}_{2} \mathrm{C}_{3}$-II. From Fig. 2, Table II and the definition of the first harmonic index, we can write directly;

$$
\begin{gathered}
H_{1}\left(S i_{2} C_{3}-I I\right)=\sum_{u v \in E\left(S i_{2} C_{3}-I I\right)} \frac{2}{d_{u}+d_{v}} \\
=2 x \frac{2}{3}+1 x \frac{2}{4}+2 x \frac{2}{4}+2 x \frac{2}{4}+\left(\begin{array}{l}
2 p \\
+2 q-4
\end{array}\right) x \frac{2}{4} \\
+(2 p+2 q) x \frac{2}{5}+(2 p+2 q-2) x \frac{2}{5}+\left(\begin{array}{l}
2 p \\
+2 q-6
\end{array}\right) \\
x \frac{2}{5}+\left(\begin{array}{l}
2 p \\
+2 q-6
\end{array}\right) x \frac{2}{5}+2 x \frac{2}{6}+\left(\begin{array}{l}
2 p \\
+2 q-8
\end{array}\right) x \frac{2}{6}+2 x \frac{2}{6} \\
+\left(\begin{array}{l}
2 p+ \\
2 q-3
\end{array}\right) x \frac{2}{6}+\left(\begin{array}{l}
2 p \\
+2 q-5
\end{array}\right) x \frac{2}{6}+\left(\begin{array}{l}
15 p q-19 p \\
-19 q+23
\end{array}\right) \times \frac{2}{6}
\end{gathered}
$$


After necessary simplifications, we get;

$$
=5+p+q+(8 p+8 q-14) x \frac{2}{5}+(15 p q-13 p-13 q+9) \times \frac{1}{3}
$$

And now, we prove the fifth harmonic index of $\mathrm{Si}_{2} \mathrm{C}_{3}$-II. From Fig. 2, Table II and the definition of the fifth harmonic index, we can write directly;

$$
\begin{aligned}
& H_{5}\left(G \mathrm{Gi}_{2} \mathrm{C}_{3}-I I\right)=\sum_{u v \in E\left(G S i_{2} C_{3}-I I\right)} \frac{2}{S_{u}+S_{v}} \\
& =2 x \frac{2}{5}+1 x \frac{2}{10}+2 x \frac{2}{7}+2 x \frac{2}{9}+(2 p+2 q-4) x \frac{2}{10} \\
& +(2 p+2 q) x \frac{2}{12}+(2 p+2 q-2) x \frac{2}{13}+(2 p+2 q-6) \\
& x \frac{2}{14}+(2 p+2 q-6) x \frac{2}{13}+2 x \frac{2}{15}+(2 p+2 q-8) x \frac{2}{16}+ \\
& 2 x \frac{2}{14}+(2 p+2 q-3) x \frac{2}{16}+(2 p+2 q-5) x \frac{2}{17} \\
& +(15 p q-19 p-19 q+23) x \frac{2}{18}
\end{aligned}
$$

After necessary simplifications, we get;

$$
\begin{aligned}
& =\frac{4}{5}+\frac{1}{5}+\frac{6}{7}+\frac{4}{9}+(2 p+2 q-4) \times \frac{1}{5}+(p+q) \times \frac{1}{3} \\
& +(2 p+2 q-2) \times \frac{2}{13}+(2 p+2 q-6) \times \frac{1}{7} \\
& +(2 p+2 q-6) \times \frac{2}{13}+\frac{4}{15}+(2 p+2 q-8) \times \frac{1}{8} \\
& +(2 p+2 q-3) \times \frac{1}{8}+(2 p+2 q-5) \times \frac{2}{17} \\
& +(15 p q-19 p-19 q+23) \times \frac{1}{9}
\end{aligned}
$$

\section{CONCLuSIONS}

In this study, we calculated the ve-ABC, ve-GA, ve- $\mathrm{H}$, and ve- $\chi$, the first harmonic and the fifth harmonic molecular topological properties of the two silicon carbide structures: $\mathrm{Si}_{2} \mathrm{C}_{3}-\mathrm{I}$ and $\mathrm{Si}_{2} \mathrm{C}_{3}$-II. These calculations enable to understand and to give information about the underlying topology of $\mathrm{Si}_{2}$ $\mathrm{C}_{3}$-I and $\mathrm{Si}_{2} \mathrm{C}_{3}$-II.

Mathematical properties of ev-degree, ve-degree, and novel harmonic topological indices are interesting studies for further studies. It is also interesting to compute ve-degree based topological indices and novel harmonic indices of naostructures and molecules for further studies.

\section{REFERENCES}

Anjum, M.S. and Safdar, M.U., 2019. K Banhatti and K hyper-Banhatti indices of nanotubes. Engineering and Applied Science Letters, 2(1), pp.19-37.

Chellali, M., Haynes, T.W., Hedetniemi, S.T. and Lewis, T.M., 2017. On vedegrees and ev-degrees in graphs. Discrete Mathematics, 340(2), pp.31-38.

Ediz, S., 2010. Computing ediz eccentric connectivity index of an infinite class of nanostar dendrimers. Optoelectronics and Advanced Materials: Rapid Communications, 4, pp.1847-1848.
Ediz, S., 2011. On the ediz eccentric connectivity index of a graph. Optoelectronics and Advanced Materials: Rapid Communications, 5(11), pp.1263-1264.

Ediz, S., 2013. The ediz eccentric connectivity index of one pentagonal carbon nanocones. Fullerenes, Nanotubes and Carbon Nanostructures, 21(2), pp. 113-116.

Ediz, S., 2017. A new tool for QSPR researches: Ev-degree randić index. Celal Bayar Journal of Science, 13(3), pp.615-618.

Ediz, S., 2017. Predicting some physicochemical properties of octane isomers: A topological approach using ev-degree and ve-degree zagreb indices. International Journal of Systems Science and Applied Mathematics, 2(5), pp.87-92.

Ediz, S., 2018. On ve-degree molecular topological properties of silicate and oxygen networks. International Journal of Computing Science and Mathematics, 9(1), pp.1-12

Ediz, S., Farahani, M.R. and Imran, M., 2017. On novel harmonic indices of certain nanotubes. International Journal of Advanced Biotechnology and Research, 8(4), pp.87-92.

Gao, W., Asif, M. and Nazeer, W., 2018. The study of honey comb derived network via topological indices. The Open Journal of Mathematical Analysis, 2(2), pp.10-26.

Imran, M., Siddiqui, M., Naeem, M. and Iqbal, M., 2018. On topological properties of symmetric chemical structures. Symmetry, 10(5), pp.173-182.

Jiang, J.W., Wang, B.S. and Park, H.S., 2018. Topologically protected interface phonons in two-dimensional nanomaterials: Hexagonal boron nitride and silicon carbide. Nanoscale, 10(29), pp.13913-13923.

Kaźmierczak-Bałata, A. and Mazur, J., 2018. Effect of carbon nanoparticle reinforcement on mechanical and thermal properties of silicon carbide ceramics. Ceramics International, 44(9), pp.10273-10280.

Kwun, Y., Virk, A., Nazeer, W., Rehman, M. and Kang, S., 2018. On the multiplicative degree-based topological indices of silicon-carbon Si2C3-I [p, q] and Si2C3-II [p, q]. Symmetry, 10(8), pp.320-336

Mei, H., Huang, W., Hua, C., Xu, Y. and Cheng, L., 2018. Manufacturing isotropic carbon fibre preforms for multilayered silicon carbide composites with a pyrolytic carbon interphase. Journal of Manufacturing Processes, 34, pp.62-69.

Munir, M., Nazeer, W., Nizami, A.R., Rafique, S. and Kang, S.M., 2016. M-polynomials and topological indices of titania nanotubes. Symmetry, 8(11), pp.117-125.

Munir, M., Nazeer, W., Rafique, S. and Kang, S.M., 2016. M-polynomial and degree-based topological indices of polyhex nanotubes. Symmetry, 8(12), pp.149-156.

Munir, M., Nazeer, W., Rafique, S. and Kang, S.M., 2016. M-polynomial and related topological indices of Nanostar dendrimers. Symmetry, 8(9), pp.97-108.

Reddy, P.S., Kesavan, R. and Ramnath, B.V., 2018. Investigation of mechanical properties of aluminium 6061-silicon carbide, boron carbide metal matrix composite. Silicon, 10(2), pp.495-502.

Sahin, B. and Ediz, S., 2018. On ev-degree and ve-degree topological indices. Iranian Journal of Mathematical Chemistry, 9(4), pp.263-277.

Seal, S., Glover, M.D. and Mantooth, H.A., 2018. 3-d wire bondless switching cell using flip-chip-bonded silicon carbide power devices. IEEE Transactions on Power Electronics, 33(10), pp.8553-8564.

Shao, Z., Virk, A.R., Javed, M.S., Rehman, M.A. and Farahani, M.R., 2019. Degree based graph invariants for the molecular graph of Bismuth Tri-Iodide. Engineering and Applied Science Letters, 2(1), pp.1-11.

Snead, L.L., Katoh, Y., Koyanagi, T. and Terrani, K., 2019. Stored energy release in neutron irradiated silicon carbide. Journal of Nuclear Materials, 514, pp.181-188.

Taguchi, T., Yamamoto, S. and Ohba, H., 2018. Ion irradiation-induced novel microstructural change in silicon carbide nanotubes. Acta Materialia, 154, pp.90-99. 
Van der Broeck, C.H., Ruppert, L.A., Lorenz, R.D. and De Doncker, R.W., 2018. Methodology for active thermal cycle reduction of power electronic modules. IEEE Transactions on Power Electronics, 34(8), pp.8213-8229.

Virk, A.R., Jhangeer, M.N., Rehman, M.A., 2018. Reverse zagreb and reverse hyper-zagreb indices for silicon carbide Si2c3-I[r,s] and Si2C3-II [r,s].
Engineering and Applied Science Letters, 1(2), pp.37-50.

Wing, B.L. and Halloran, J.W., 2018. Relaxation of residual microstress in reaction bonded silicon carbide. Ceramics International, 44(10), pp.11745-11750.

Zhong, L., 2012. The harmonic index for graphs. Applied Mathematics Letters, 25, pp.561-566. 